\title{
"It's Like Crossing a Bridge" Complexities Preventing Physicians from Discussing Deactivation of Implantable Defibrillators at the End of Life
}

\author{
Nathan E. Goldstein, $M D^{1,2}$, Davendra Mehta, $M D P h D^{3}$, Ezra Teitelbaum, $B A^{7}$, \\ Elizabeth H. Bradley, $\mathrm{PhD}^{4}$, and R. Sean Morrison, $\mathrm{MD}^{1,2}$
}

'Department of Geriatrics Mount Sinai School of Medicine, One Gustave Levy Place, Box 1070, New York, NY 10029, USA; ${ }^{2}$ Geriatric Research Education and Clinical Care Center, James J. Peters Veterans Affairs Health System, Bronx, NY, USA; ${ }^{3}$ Department of Medicine Mount Sinai School of Medicine, New York, NY, USA; ${ }^{4}$ Department of Epidemiology and Public Health and Robert Wood Johnson Clinical Scholars Program, Yale, School of Medicine, New Haven, CT, USA.

OBJECTIVE: To understand potential barriers to physician-initiated discussions about Implantable Cardioverter Defibrillator (ICD) deactivation in patients with advanced illness.

DESIGN: Qualitative one-on-one interviews.

PARTICIPANTS: Four electrophysiologists, 4 cardiologists, and 4 generalists (internists and geriatricians) from 3 states.

APPROACH: Clinicians were interviewed using openended questions to elicit their past experiences with discussing deactivating ICDs and to determine what barriers might impede these discussions. Transcripts of these interviews were analyzed using the qualitative method of constant comparison.

RESULTS: Although many physicians believed that conversations about deactivating ICDs should be included in advance care planning discussions, they acknowledged that they rarely did this. Physicians indicated that there was something intrinsic to the nature of these devices that makes it inherently difficult to think of them in the same context as other management decisions at the end of a patient's life. Other explanations physicians gave as to why they did not engage in conversations included: the small internal nature of these devices and hence absence of a physical reminder to discuss the ICD, the absence of an established relationship with the patient, and their own general concerns relating to withdrawing care.

CONCLUSION: Whereas some of the barriers to discussing ICD deactivation are common to all forms of advance care planning, ICDs have unique characteristics that make these conversations more difficult. Future educational interventions will need to be designed to teach physicians how to improve communication with patients about the management of ICDs at the end of life.

Data from this study were presented at the 2006 national meeting of the American Association of Hospice and Palliative Medicine (AAHPM).
KEY WORDS: palliative care; advanced technology; communication; patient-physician relationship; implantable cardioverter defibrillator. J Gen Intern Med 23(Suppl 1):2-6 DOI: $10.1007 / \mathrm{s} 11606-007-0237-\mathrm{x}$

(C) Society of General Internal Medicine 2007

\section{INTRODUCTION}

There is a large body of evidence demonstrating that Implantable Cardioverter Defibrillators (ICDs) decrease the incidence of sudden cardiac death, ${ }^{1-4}$ but little is known about the endof-life care for these patients, most of whom die from either worsening of their underlying heart disease or other chronic illnesses. As a patient's condition deteriorates, physiologic changes (intrinsic and extrinsic to the heart) may affect the cardiac conduction system, leading to more arrhythmias and increasing the frequency of shocks. One study showed that up to $20 \%$ of family members of deceased patients reported that their loved one received a shock from their ICD in the last days to minutes of life. ${ }^{5}$ Because ICD shocks can cause pain and anxiety and may not prolong a life of acceptable quality, ${ }^{6-8}$ it is appropriate to consider ICD deactivation as a patient's clinical status worsens and death is near. Previous work has shown that clinicians and patients rarely engage in discussions about deactivating ICDs and most remain active until death. ${ }^{5}$

Given the expanding indications for ICD implantation, ${ }^{1-4,9,10}$ the issue of device deactivation will become more relevant as the population ages. Under current Medicare criteria, approximately 3-4 million patients are currently eligible to receive these devices, with 400,000 new patients eligible each year. ${ }^{3,11-13}$ Prior research has suggested that patients prefer physicians to initiate discussions about treatment options at the end of life. As most patients with an ICD will face a decision about deactivating the device near the end of their lives, we conducted this study to better understand barriers to clinicianinitiated discussions about ICD deactivation. Because these conversations are infrequent and complex, it would be difficult to begin exploring the barriers impeding deactivation discussions using a traditional, closed-ended survey instrument. Instead, the qualitative method of using open-ended questions to conduct initial explorations ${ }^{14-16}$ is more suited for understanding how physicians conceptualize the role of the device 
and determining barriers that impede engaging in conversations with patients about deactivating ICDs.

\section{METHODS}

\section{Study Design and Data Collection}

We conducted a qualitative study using in-depth interviews ${ }^{17,18}$ of physicians by means of open-ended questions from a predetermined discussion guide (Table 1). The questions for the guide were determined by consensus among several of the investigators (NG, EB, RSM) using both their clinical experiences with this issue and their expertise in qualitative methods. The guide begins with having physicians describe how they view their individual role in the overall care of patients with ICDs and how they explain to patients that they need an ICD. (These data are not reported in this manuscript.) Next, physicians are asked to describe a particularly memorable experience(s) relating to ICD deactivation discussions. (Of note, many ICDs are multifunctional devices that may also perform a pacing or resynchronization function. For purposes of this discussion, the term deactivation only refers to turning off the shocking function of a defibrillator). During the course of describing this conversation, physicians were probed to determine what difficulties they encountered during these conversations. Finally, the interviewer described to the participants the medical literature revealing the low prevalence of these discussion in routine care ${ }^{5}$ and asked clinicians why they thought these discussions happen so rarely.

Although many clinicians may care for patients near the end of life with ICDs, the investigators chose to focus on primary care physicians and those specialists who would be most involved with a patient's ICD. Therefore, we enrolled 4 groups of clinicians: general internists, geriatricians, cardiologists, and electrophysiologists. We believed that the most efficient way to determine barriers to these conversations was to interview clinicians who have had these discussions, so we only enrolled physicians who previously had a conversation about deactivation. In addition, physicians had to be in

\section{Table 1. Question Guide For In-Depth, Open-ended Interviews With} Clinicians

\footnotetext{
Questions

1. Explain to me how you see your role as a clinician in terms of the overall health of the patient.

-Probe to determine how they see their role in terms of guiding medical care/patient decision making.

-Probe to see if doctors see themselves as primary care provider for patients especially for those with ICDs.

2. Tell me how you explain to patients that they need an ICD

-Probe to see if information about prognosis, life-expectancy, and deactivation is given at time of implantation.

3 . We are trying to understand more about situations in which doctors might consider deactivating ICDs. Have you ever been involved in the care of a patient who needed a device deactivated? Tell me about your experience.

-Probe both for experience discussing deactivating and experience with actually turning devices off.

4. Previous research in this issue by our team has found that conversations about deactivating ICDs in patients with advanced illness rarely happens. Why do you think this might be?

-Probe to ask if these conversations are different than others at the end of life.

5. Is there anything else I should ask so I can better understand how you deal with terminally ill/dying patients and their ICDs?
}

practice at least 1 year. To identify eligible physicians, the investigators sent out "broadcast" emails to the divisions of each of these 4 specialties at major academic medical centers throughout the greater New York City area (including New Jersey and Connecticut); personally contacted individual physicians whom they thought might have had such discussions (e.g., physicians specializing in palliative care, cardiologists with a primarily geriatric population); and asked physicians participating in the study to identify other potential clinicians whom they thought might have had these conversations. We were not able to identify family physicians who had these discussions, and this may be because of the limited role these clinicians play in the New York City metro area.

The investigators used purposeful sampling to balance the number of physicians of each type that were enrolled in the study. The research protocol was approved by the institutional review board of the principal investigator's medical school, and all physicians signed informed consent. All physicians were interviewed by the primary investigator (NG) either in person in their office or via telephone. All interviews were audio-taped and subsequently transcribed.

\section{Data Analysis}

We used the constant comparative method ${ }^{18,19}$ of qualitative data analysis to develop and implement consistent and comprehensive coding of the open-ended data. First, the data were reviewed line-by-line until a concept became apparent at which time a code was assigned. As more data were reviewed, the code was further refined. Subsequent transcripts were then analyzed and compared to the list of codes to ascertain whether they reflected the same concepts. ${ }^{20}$ New codes were added as needed until no new concepts emerged with successive interviews (i.e., thematic saturation). All transcripts were independently reviewed and coded by 3 of the reviewers (NG, ET, RSM) who met to discuss the interviews and the coding structure on a regular basis. Discrepancies occurred rarely ( $<5$ times), and related more to refining themes than to determining to which category a particular passage should be assigned. In cases of disagreement, the 3 investigators came to a negotiated consensus through further review and discussion of the context and content, as recommended by experts in qualitative analysis. ${ }^{21}$ The group determined by consensus at which point no new themes emerged from the data. At this time, it was decided that thematic saturation had been reached and thus the data collection period was closed.

\section{RESULTS}

Thematic saturation was achieved after 12 participant interviews, and their characteristics are shown in Table 2.

When asked, almost every physician agreed that conversations about ICD deactivation should occur, but they all acknowledged that they rarely did this. Clinicians understood the importance of discussing ICD deactivation, but had difficulty translating the theoretical importance of these conversations to real-life scenarios. As described by a female Internist:

It hadn't occurred to me to turn it off until [the cardiology fellow said] you could turn these things off and I'm like, 'oh, okay.' I mean it wasn't something that I had ever encountered, and it crossed my mind on a technical level, but not really, 'oh, I should have this conversation. 
Table 2. Demographics of Physician Participants

\begin{tabular}{ll}
\hline \hline Characteristics & \\
\hline Age & \\
Range & $33-61$ \\
Median (SD) & 36.5 (9.2) \\
Years since completing training & \\
Range & $1-29$ \\
Median (SD) & $3.5(9.2)$ \\
Gender & \\
Female & 7 \\
Specialty & \\
Cardiology & 4 \\
Electrophysiology & 4 \\
Generalist(generalists evenly & 4 \\
split between Internists and & \\
geriatricians) & \\
Ethnicity & \\
White & \\
Asian & \\
Practice Setting & \\
Hospital-Associated & 4 \\
Private & \\
Location & \\
New York & \\
New Jersey & \\
Connecticut & 10 \\
\hline
\end{tabular}

When asked why they had such difficulty engaging in these conversations, participants postulated that there was something intrinsic to the nature and function of these devices that made it inherently difficult to think of them in the same context as other management decisions at the end of a patient's life. One physician described this as follows:

People go on and off medications all the time; I put someone on Lasix, I increase their Lasix, I stop their Lasix, no big deal. You can change it again and again, but turning off a [defibrillator] is; it's like crossing a bridge to a certain extent. It's saying, 'you know, we have gone as far as we can go and you're not going to need this down the road you're traveling and we're going to shut it off.' There's a finality to turning it off that is not the same as just saying, well we'll stop this medication [and then later] we can restart it, because if you've made the decision to stop...the surveillance of a defibrillator, if you've made it for the right reasons, you're not going to be turning it back on."-male cardiologist

Another cardiologist suggested that these conversations are difficult to include in the context of other conversations because of the primary life-saving role of these devices. As she described:

When you start talking about ... turning it off, then you are sort of shutting off the hope.

In addition to its overall role, the small, unseen nature of the device makes it difficult for physicians to remember to include them as part of larger discussions about advance care planning, as characterized by a female cardiologist who stated:

I think that one thing is that people don't think about [turning it off] because it's internalized.
Whereas some clinicians thought that the differences between ICDs and other technologies (e.g., ventilators, dialysis) used at the end of life make conversations about deactivation more difficult, other clinicians also believed that certain characteristics unique to the ICD make deactivation conversations easier.

Well, I think it's different than a respirator, for example, because it's, you know, it's not like you turn [the ICD] off and the person dies."-female electrophysiologist

Another female electrophysiologist described this phenomenon similarly when she said,

Well, I don't know, I think it makes it easier because it's sort of a random event in a sense. That, you know, by turning off the switch you're not killing the person.

Difficulties in including ICD deactivation discussions into other forms of care planning are not limited to the nature of the device itself. Another consistent theme was that physicians (both generalists and specialists) lacked the sense of rapport with patients to be able to discuss ICD deactivation. As a female electrophysiologist stated:

I think you have to develop some sort of rapport with the patient before you can start to discuss [ICD deactivation]. For one thing, you don't want to scare the patient the first time you see them into thinking that they're dying.

Another clinician also described the importance of an established relationship by stating:

I think most doctors don't do [ICD deactivation discussions] well. I think it is because they don't have the time ... they don't know the patients well enough because of the way you practice now with insurance, you know, you don't develop the bond with patients now the way you used to."-female cardiologist

A final barrier to communicating about ICD deactivation was a feeling that deactivation was withdrawing life-sustaining care. As a clinician stated:

I think that people just don't think of turning off things that were already started, even though it's like all technology, even though we say ethically and legally that there's no difference between withholding and withdrawing, I think for a lot of life-sustaining therapies, in practice it seems like it's different."-female internist

\section{DISCUSSION}

Results from these qualitative interviews with clinicians from a variety of specialties in both academic and private practice settings demonstrate that although these physicians believe conversations about deactivating ICDs should happen as part of the other advance care planning discussions for patients with serious illness, this rarely occurs. Some of the reasons why these conversations do not occur seem to be similar to the 
larger literature on advance care planning (i.e., those discussions where patients' and their families' goals of care are elicited and treatment plans adjusted to be in line with those goals $^{22}$ ), whereas others are unique to the ICD.

Many physicians in this study felt that because of a lack of time, they did not have a relationship with patients that would facilitate discussions about ICD deactivation-a finding other investigators have noted when examining barriers to advance care planning for patients with a variety of illnesses. ${ }^{23,24}$ Clinicians also noted that they did not "do discussions well" and were concerned that they would either take away patients' hope or raise concerns about death; these are all concepts that have been found in other studies exploring barriers to advance care planning. ${ }^{23-26}$ Physicians also discussed general concerns with withdrawing therapies. Whereas discussion about the withholding or withdrawing treatments are considered the same by ethicists, ${ }^{27}$ it is known that physicians draw a distinction between these 2 treatments. ${ }^{28-30}$

There were issues relating to ICD deactivation, however, that were unique to the nature of the device itself. The majority of patients who have hemodialysis or ventilators withdrawn (the two most frequently studied technologies that are commonly withdrawn, ${ }^{31-37}$ ) will often die within hours to weeks. The interval between ICD deactivation and patient death may be much longer given the unpredictable nature of malignant arrhythmias. Because of this distinction between ICDs and other advanced technologies, clinicians who engage in conversations about deactivation are somehow insulated from feeling that they are "killing the person." Both hemodialysis and ventilator support also require large machinery, which creates a physical reminder that advanced technologies are being used to sustain life. In addition, these interventions have a large impact on the patient's overall quality of life. Because the ICD is so small and innocuous, its size does not create a daily interference with a patient's quality of life; therefore, discussions about their management at the end of life may not seem so pressing to clinicians. It would seem as though the nature of the device makes it less noticeable, but its small physical size seems to be not correlated to the large ethical dilemma it creates for physicians.

It is noteworthy that despite the difficulties added by the unique nature of the ICD, every clinician who participated in this study (whether in the role of primary care provider or specialist) felt they had a role in these conversations. Traditionally, one might assume that the job of having "difficult conversations" is that of the primary care provider, but there were no participants who expressed that they should be excluded from these conversations based on their medical subspecialty.

These data make it clear that clinicians believe they should engage in conversations about ICD deactivation, but the difficulty lies in creating effective future interventions to make these conversations easier. These conversations are made even more complex by the fact that in qualitative studies with patients (reported separately in this issue of the Journal) we found that relatively healthy community-dwelling outpatients with ICDs may not wish to engage in conversations regarding ICD deactivation. Although educational interventions have shown significant improvements in facilitating communication between clinicians and patients with advanced illness, ${ }^{38-40}$ the data reported here demonstrate that there are unique barriers to communication about ICD deactivation, which might benefit from future interventions specifically designed for conversations about the management of these devices. These tools will need to take into account patients' reluctance to engage in these conversations, as well as the unique nature of the ICD and how this might hinder conversation.

As the number of implanted devices continues to expand in the future because of expanding eligibility and reimbursement, these conversations will be encountered with more frequency. Only by assuring that conversations about ICD deactivation routinely occur for all patients with advanced illness can we assure that patients and their families have the highest quality of care near the end of life.

Acknowledgments: This research is supported by Dr. Nathan Goldstein's Hartford Geriatrics Health Outcomes Research Scholars Award, his Hartford Center of Excellence Scholar Award, and his Mentored Patient-oriented Research Career Development Award (1 K23 AG025933-01A1). Mr. Teitelbaum was supported by the American Federation for Aging Research Summer Medical Student Research Program. Dr. Bradley is supported by the Patrick and Catherine Weldon Donaghue Medical Research Foundation Investigator Award. Dr. Morrison is supported by a Midcareer Investigator Award in patient-oriented research award (1K24AG22345-01) from the National Institute on Aging and the National Palliative Care Research Center.

Conflict of Interest: The second author has previously received support from Medtronic, Inc., Academic Pharmaceuticals, Inc., Procter and Gamble Pharmaceuticals, Inc., and Statistics and Epidemiology Research Corporation, but this author was not receiving these funds during the course of this project. The other authors have no conflict of interest to disclose.

Corresponding Author: Nathan E. Goldstein MD, Department of Geriatrics Mount Sinai School of Medicine, One Gustave Levy Place, Box 1070, New York, NY 10029, USA (e-mail: Nathan. Goldstein@mssm.edu).

\section{REFERENCES}

1. Buxton AE, Lee KL, Fisher JD, Josephson ME, Prytowsky EN, Hafley GA. Randomized study of the prevention of sudden death in patients with coronary artery disease. N Engl J Med 1993;341(25):1882-90.

2. Moss AJ, Hall WJ, Cannom DS, et al. Improved survival with an implanted defibrillator in patients with coronary disease at high risk for ventricular arrhythmia. N Engl J Med. 1996;335(26):1933-40.

3. Moss AJ, Zareba W, Hall WJ, et al. Prophylactic implantation of a defibrillator in patients with myocardial infarction and reduced ejection fraction. N Engl J Med. 2002;346(12):877-83.

4. Bardy GH, Lee KL, Mark DB, et al. Amiodarone or an implantable cardioverter-defibrillator for congestive heart failure. N Engl J Med. 2005;352(3):225-37.

5. Goldstein NE, Lampert R, Bradley EH, Lynn J, Krumholz HM. Management of implantable cardioverter defibrillators in end-of-life care. Ann Intern Med. 2004; 141:835-38.

6. Glikson M, Friedman PA. The implantable cardioverter defibrillator. Lancet. 2001;357:1107-17.

7. Eckert M, Jones T. How does an implantable cardioverter defibrillator (ICD) affect the lives of patients and their families? Int J Nurs Pract. 2002;8:152-57.

8. Sears SF, Conti J. Quality of life and psychological functioning of ICD patients. Heart. 2002;87:488-93.

9. Kadish A, Dyer A, Daubert JP, et al. Prophylactic defibrillator implantation in patients with nonischemic dilated cardiomyopathy. $\mathrm{N}$ Engl J Med. 2004;350(21):2151-8.

10. Bristow MR, Saxon LA, Boehmer J, et al. Cardiac-resynchronization therapy with or without an implantable defibrillator in advanced chronic heart failure. N Engl J Med. 2004;350(21):2140-50.

11. Gillick MR. Medicare coverage for technological innovations-time for new criteria? N Engl J Med. 2004;350(21):2199-203. 
12. Brown D. Medicare to cover heart devices. The Washington Post 2003 June 7;A04.

13. Hlatky MA, Sanders GD, Owens DK. Evidence-based medicine and policy: the case of the implantable cardioverter defibrillator. Health Aff (Millwood). 2005;24(1):42-51.

14. Sofaer S. Qualitative methods: what are they and why use them? Health Serv Res. 1999;34(5 Pt 2):1101-18.

15. Sofaer S. Qualitative research methods. Int J Qual Health Care. 2002; 14 (4):329-36.

16. Strauss A, Corbin JM. Basics of Qualitative Research: Techniques and Procedures for Developing Grounded Theory. Thousand Oaks: SAGE Publications, Inc.; 1998.

17. McCracken G. The Long Interview. Thousand Oaks, CA: Sage Publications; 1988.

18. Strauss A, Corbin JM. Basics of Qualitative Research: Grounded Theory Procedures and Techniques. 2nd Edition. San Francisco: Sage Publications; 1990.

19. Glaser B, Strauss A. The Discovery of Grounded Theory: Strategies for Qualitative Research Chicago. Aldine Publishing Company; 1967.

20. Bradley EH, Curry L, Devers K. Qualitative data for health services research: developing taxonomy, themes, and theory. Health Serv Res. 2007. DOI: $10.1111 /$ j.1475-6773.2006.00684.x

21. Patton MQ. Qualitative Research and Evaluation Methods. San Francisco: Sage Publications; 2001.

22. Lynn J, Goldstein NE. Advance care planning for fatal chronic illness: avoiding commonplace errors and unwarranted suffering. Ann Intern Med. 2003;138(10):812-8.

23. Morrison RS, Morrison EW, Glickman DF. Physician reluctance to discuss advance directives. An empiric investigation of potential barriers. Arch Intern Med. 1994;154(20):2311-8.

24. Curtis JR. Communicating about end-of-life care with patients and families in the intensive care unit. Crit Care Clin. 2004;20(3):363-80.

25. Weiner JS, Cole SA. Three principles to improve clinician communication for advance care planning: overcoming emotional, cognitive, and skill barriers. J Palliat Med. 2004;7(6):817-29.

26. Perry E, Swartz R, Smith-Wheelock L, Westbrook J, Buck C. Why is it difficult for staff to discuss advance directives with chronic dialysis patients? J Am Soc Nephrol. 1996;7(10):2160-8.

27. Luce JM, Alpers A. Legal aspects of withholding and withdrawing life support from critically ill patients in the United States and providing palliative care to them. Am J Respir Crit Care Med. 2000;162:2029-32.
28. Faber-Langendoen K, Bartels DM. Process of forgoing life-sustaining treatment in a university hospital: an empirical study. Crit Care Med. 1992;20(5):570-7.

29. Farber NJ, Simpson P, Salam T, Collier VU, Weiner J, Boyer EG. Physicians' decisions to withhold and withdraw life-sustaining treatment. Arch Intern Med. 2006;166(5):560-4.

30. Solomon MZ, O'Donnell L, Jennings B, et al. Decisions near the end of life: professional views on life-sustaining treatments. Am J Public Health 1993;83(1):14-23.

31. Holley JL, Foulks CJ, Moss AH. Nephrologists' reported attitudes about factors influencing recommendations to initiate or withdraw dialysis. J Am Soc Nephrol. 1991;1(12):1284-8.

32. Hamel MB, Davis RB, Teno JM, et al. Older age, aggressiveness of care, and survival for seriously ill, hospitalized adults. SUPPORT Investigators. Study to Understand Prognoses and Preferences for Outcomes and Risks of Treatments. Ann Intern Med. 1999;131(10):721-8.

33. Cohen LM, Germain MJ, Poppel DM. Practical considerations in dialysis withdrawal: "to have that option is a blessing". JAMA 2003;289(16):2113-9.

34. Phillips RS, Hamel MB, Teno JM, et al. Patient race and decisions to withhold or withdraw life-sustaining treatments for seriously ill hospitalized adults. SUPPORT investigators. study to understand prognoses and preferences for outcomes and risks of treatments. Am J Med. 2000;108 (1):14-9.

35. Marr L, Weissman DE. Withdrawal of ventilatory support from the dying adult patient. J Support Oncol. 2004;2(3):283-8.

36. Rubenfeld GD. Principles and practice of withdrawing life-sustaining treatments. Crit Care Clin. 2004;20(3):435-51, ix

37. Cook D, Rocker G, Marshall J, et al. Withdrawal of mechanical ventilation in anticipation of death in the intensive care unit. N Engl $\mathrm{J}$ Med. 2003;349(12):1123-32.

38. Robinson $\mathbf{K}$, Sutton S, von Gunten CF, et al. Assessment of the Education for Physicians on End-of-Life Care (EPEC) Project. J Palliat Med. 2004;7(5):637-45.

39. Weiner JS, Arnold RM, Curtis JR, Back AL, Rounsaville B, Tulsky JA Manualized communication interventions to enhance palliative care research and training: rigorous, testable approaches. J Palliat Med. 2006;9(2):371-81.

40. Back AL, Arnold RM, Baile WF, Tulsky JA, Fryer-Edwards K. Approaching difficult communication tasks in oncology. CA Cancer $\mathrm{J}$ Clin. 2005;55(3): 164-77. 